\title{
Cavuldak, Ahmet (Hrsg.) (2020): Peter Graf Kielmansegg im Gespräch. Übungen im politischen Denken
}

\author{
Baden-Baden: Nomos. 404 Seiten. 39€
}

\section{Tine Stein}

Angenommen: 23. September 2021 / Online publiziert: 13. Oktober 2021

(C) Der/die Autor(en) 2021

Das wissenschaftsbiografische Interview ist ein Genre, in dem in den vergangenen Jahren einige höchst lesenswerte Bücher entstanden sind. Ernst-Wolfgang Böckenförde, Dieter Grimm und Fritz W. Scharpf haben über ihren familiären Hintergrund, die Zeit des Studiums, ihr wissenschaftliches Werk, ihr öffentliches Wirken und nicht zuletzt über ihre Erfahrungen ,nach der Katastrophe“ Auskunft gegeben: als diejenigen, die den Nationalsozialismus und den Zweiten Weltkrieg als Kinder erlebten, dann den Aufbau der Bundesrepublik als Heranwachsende, die Studentenunruhen Ende der 1960er-Jahre, die deutsche Teilung und die Wiedervereinigung, die europäische Integration, die Finanzkrise, um nur einige der wichtigsten historischen Etappen zu nennen. Nun liegt ein weiterer, von Ahmet Cavuldak klug komponierter edierter Interviewband mit einem Vertreter dieser generativen Kohorte vor, der zudem 16 sorgfältig ausgesuchte Aufsätze und Essays des Interviewten enthält.

Peter Graf Kielmansegg, Jahrgang 1937, ist ein Solitär in der deutschen Politikwissenschaft. Als Professor für Politikwissenschaft von 1971-1985 zunächst in Köln, dann bis zu seiner Emeritierung im Jahr 2002 in Mannheim lehrend, danach unter anderem als Präsident der Heidelberger Akademie der Wissenschaften und Vizepräsident der Studienstiftung tätig, hat er weder eine Schule begründet noch Verbundforschungsprojekte geleitet, Bücher und Aufsätze von ihm sind durchweg auf Deutsch veröffentlicht. Seine Autorität auch über die Grenzen der Politikwissenschaft hinaus ist aber heute, wo die Kriterien drittmittelfinanzierter Forschung und internationaler Sichtbarkeit bei Berufungen entscheidend sind, unbestritten und weithin anerkannt. Das war nicht immer so. Einer vertieften Rezeption stand nicht so sehr seine liberalkonservative Haltung als solche im Wege, als vielmehr die Grundthese der Vorzugswürdigkeit des demokratischen Verfassungsstaates, den er als die

Tine Stein $(\bowtie)$

Georg-August-Universität Göttingen, Göttingen, Deutschland

E-Mail: tine.stein@uni-goettingen.de 
angemessene Legitimitätsgrundlage politischer Herrschaft unter den normativen Vorzeichen gleicher Freiheit und der Orientierung der Regelung der öffentlichen Angelegenheit am Gemeinwohl ansieht. Kritik als Infragestellung trumpfte aber lange Zeit als vermeintlich wissenschaftlicher und auch normativ anspruchsvoller über Rekonstruktion, die im Ruf von Apologie und Affirmation des Bestehenden stand. Dabei liegt Kielmansegg nichts so fern wie eine idealisierende Betrachtung des demokratischen Verfassungsstaates. In seinen Schriften kommen drei Qualitäten zur Geltung, denen sich seine Autorität als Gelehrter verdankt: erstens eine analytische und begriffliche Tiefenschärfe, mit der Kielmansegg seine Untersuchungsgegenstände rund um die Legitimitätsgrundlagen des demokratischen Verfassungsstaates demokratietheoretisch durchleuchtet, wobei er ideengeschichtliche und historische Analyse mit systematischer Reflexion verbindet; zweitens eine Treffsicherheit und Ausgewogenheit des Urteils, das man immer erhellend und konzise argumentiert findet, auch wenn man ihm im Ergebnis einmal nicht zustimmt; und drittens eine ihresgleichen suchende Klarheit und Eleganz des sprachlichen Ausdrucks, der den denkbar entferntesten Punkt gegenüber dem wechselnden sozialwissenschaftlichen Modejargon bildet. Alle drei Qualitäten kommen auch in diesem Buch zum Tragen.

Anhand vier ideengeschichtlicher Profile zu Kant, den Federalist Papers, Tocqueville und einem bislang unveröffentlichten Text zu Arendt lässt sich studieren, wie man auf knappem Raum die zentralen Aussagen eines Autors respektive einer Autorin mit einer Haltung hermeneutischer Benevolenz präsentiert und sich gleichwohl kritisch mit diesen auseinandersetzen kann. Arendts harsche Ablehnung der Repräsentativdemokratie und ihr Lob der Rätedemokratie - beides in den Augen Kielmanseggs historisch gänzlich uninformiert - und vor allem Arendts Idee von Politik als im Grunde genommen permanenter Revolution, als einer „Hoch-Zeit““ der Gründung für die Mitglieder eines Gemeinwesens, die über die Grundfragen des Zusammenlebens sprechen und entscheiden: Das alles erscheint Kielmansegg nicht gut begründet, überemphatisch und inhaltlich leer. Denn wenn das Alltagsgeschäft der Politik: Die Interessenvertretung, das Ringen um die sachlich angemessenste Lösung und die Regelung der öffentlichen Angelegenheiten für den Normalzustand aus dem eigentlichen Bereich der Politik herausdefiniert und den Expertinnen und Experten überlassen wird, zumal in Verbindung mit dem Vorrang des Öffentlichen vor dem Privaten als einem aristokratischem Ideal, ,in dem Sinne, daß es eine Lebensführungsmaxime nur für wenige ist und sein kann“" (S. 222), wie Kielmansegg kritisch anmerkt, dann sei das keine wirklich überzeugende Option für moderne Gesellschaften.

Und auch eine zeitdiagnostische Kraft ist diesem Autor eigen, der die Herausforderungen der Gegenwart in der Digitalisierung als grundlegend neue und riskante Bedingung für demokratische Willensbildung erkennt, in der Umweltkrise als Gefährdung der natürlichen Lebensgrundlagen des Menschen eine Anfrage an die auf Gegenwart fixierte Problemlösungsfähigkeit der Demokratie sieht und mit der Humangenetik eine mögliche und denkbar folgenreiche Veränderung des menschlichen Selbst- und Weltverhältnisses befürchtet. In einigen der hier abgedruckten öffentlichen Interventionen Kielmanseggs, die in den letzten Jahren als Essays in der FAZ veröffentlicht wurden, bilden diese Herausforderungen als Problemanalyse den Hintergrund. Einfache Schlussfolgerungen zieht der Autor daraus keineswegs - gerade 
in seinem letzten Essay „Politik und Apokalypse“ von 2019 wirbt er bei allem Grund für Skepsis am Ende doch hoffnungsvoll für die Chancen der Demokratie und die sich in der Krise offenbarende Kraft der Vernunft. „So halte ich fest am Prinzip Hoffnung“" (S. 124) - eine Grundhaltung, die er auch aus seinem christlichen Glauben schöpft (S. 20-21).

Die Demokratie braucht das Gespräch, damit sich die Einsicht der vielen in die Notwendigkeit der vernünftigen Lösung durchsetzen kann. Und auch der wissenschaftliche Austausch braucht das Gespräch, das analoge Gespräch in Präsenz und nicht nur im Kachelformat. Um die Zeit bis zum „,neuen Normal“ zu überbrücken, aber auch danach ist wenigstens die Teilnahme durch Lektüre eines solchen Gesprächs, hier auf 150 Seiten zwischen Graf Kielmansegg und Ahmet Cavuldak über die Generationen hinweg dargeboten, sehr zu empfehlen, zumal wenn es von einer so wohltuenden Freundlichkeit, Bescheidenheit und Offenheit seitens des im Mittelpunkt stehenden Interviewten geprägt ist. Es werden nicht nur die wichtigsten wissenschaftlichen Publikationen (auch die historischen) im Gespräch vertieft und immer wieder durch die kundigen Fragen und hilfreichen Kommentare des Herausgebers eingeordnet, sondern es werden auch die menschlichen Beziehungen deutlich, die im Fall Kielmanseggs das wissenschaftliche Gespräch haben tragen geholfen und die auch das wissenschaftliche Arbeiten ermöglichten. Darunter die Beziehung zu drei Weggefährten, denen er jeweils eine hier nachzulesende Porträtskizze gewidmet hat: zu dem Holocaust-Überlebenden Eugen Kogon, einem der ersten Lehrstuhlinhaber für Politikwissenschaft in Darmstadt, bei dem Kielmansegg in sieben freien Assistentenjahren seine historische Dissertation vollenden, zwei große neue Bücher schreiben konnte und mit dem ihm am Ende eine Freundschaft verband; dann das Gespräch mit Dolf Sternberger, von dem Kielmansegg stilistisch und ideengeschichtlich viele Anregungen erfahren hat; und auch die Verbindung zum leidenschaftlichen und immer anregenden Wilhelm Hennis ist sehr einprägsam. Hennis hat sich bekanntlich mit vielen im Fach überworfen, aber nicht mit Kielmansegg. Was sagt es aus über jemand, dass man sich wünscht, wie Hennis es für Kielmansegg schriftlich hinterlassen hat, er möge auf der eigenen Trauerfeier sprechen? Wohl vor allen Dingen, dass diesem Menschen unbedingtes Vertrauen entgegengebracht wird, dass er den richtigen Ton trifft, sozusagen mit der Autorität des letzten Wortes sprechen kann. Aus dieser Autorität heraus hat uns Kielmansegg neben all den wertvollen fachlichen Einsichten auch einen zutiefst menschlichen Ratschlag mit auf den Weg gegeben, mit dem das Interview endet und den man im Zeichen der Endlichkeit, die auch plötzlich in Beziehungen eintreten kann, sehr beherzigen sollte: dass es darauf ankommt, wie man sich voneinander verabschiedet.

Funding Open Access funding enabled and organized by Projekt DEAL.

Open Access Dieser Artikel wird unter der Creative Commons Namensnennung 4.0 International Lizenz veröffentlicht, welche die Nutzung, Vervielfältigung, Bearbeitung, Verbreitung und Wiedergabe in jeglichem Medium und Format erlaubt, sofern Sie den/die ursprünglichen Autor(en) und die Quelle ordnungsgemäß nennen, einen Link zur Creative Commons Lizenz beifügen und angeben, ob Änderungen vorgenommen wurden. 
Die in diesem Artikel enthaltenen Bilder und sonstiges Drittmaterial unterliegen ebenfalls der genannten Creative Commons Lizenz, sofern sich aus der Abbildungslegende nichts anderes ergibt. Sofern das betreffende Material nicht unter der genannten Creative Commons Lizenz steht und die betreffende Handlung nicht nach gesetzlichen Vorschriften erlaubt ist, ist für die oben aufgeführten Weiterverwendungen des Materials die Einwilligung des jeweiligen Rechteinhabers einzuholen.

Weitere Details zur Lizenz entnehmen Sie bitte der Lizenzinformation auf http://creativecommons.org/ licenses/by/4.0/deed.de. 